\title{
Effects of exercise intervention on the functional fitness of community elderly
}

\author{
Tsui-Er Lee ${ }^{a}$
}

Asia University, Office of Physical Education, 41354 Taichung City, Taiwan

\begin{abstract}
This study aims to discuss the effects of the 12-week exercise intervention on the functional fitness of community elderly. Total 25 community elderly aged above 65 are proceeded functional fitness tests. The data are analyzed with Descriptive Statistics. The results show that the male elderly significantly enhance the upper limb strength (musculus biceps brachii left arm curling, musculus biceps brachii right arm curling), static balance (openeye single leg standing), and agility and dynamic balance (chair stand detour) and the female elderly make remarkable progress on the upper limb strength (musculus biceps brachii left arm curling, musculus biceps brachii right arm curling), cardiorespiratory endurance (high knees in place), static balance (open-eye single leg standing), and agility and dynamic balance (chair stand detour) after the 12-week regular exercise intervention. With functional fitness tests, the research findings reveal that regular exercise presents the effectiveness on distinct physical fitness of community elderly, while the aerobic endurance and upper/lower body flexibility still require improvement. Apparently, it is suggested to practice courses with stages and match fitness test results to adjust types of exercise and exercise intensity in order to achieve the effect. Keywords: regular exercise, the elderly, type of exercise.
\end{abstract}

\section{Introduction}

Population is a primary element of a nation, and the number of population and the change of age structure are the key factors in the national development. Population of young adults aged 15-64 (also called working-age population) achieved the peak in 2015 and started to decrease in 2016. Furthermore, aged population would exceed young population in 2016 because of decreasing young population and increasing aged population. In comparison with 2014, the Population Projection result revealed that young population and population of young adults would reduce about a half by 2061 (52.2\% and $47.9 \%$, respectively), while aged population would increase 1.6 times [1]. It proves that the constant increase of aged population structure has aging become an inevitable trend in Taiwan.

The issues related to the elderly and health conditions therefore should be emphasized. The physical fitness and physiological function, including nerves, muscles, skeleton, cardiopulmonary function, and hormone regulation function, of humans after 25 years old would degenerate with increasing ages [2]. Such a process would continue to the death and is irreversible. Degeneration might result in decreasing activity of the elderly to affect the daily activity and cause inconvenient life. A lot of research indicated that maintaining the habit of moderate, non-exciting, and low-intensity regular exercise could enhance strengths and promote physical function, like cardiopulmonary function [3]. Aiming at the elderly aged 55-75, Pei-chen Chung \& Fen-yu Tseng preceded the 8-week exercise intervention and found out the obvious improvement of lower body strength, upper body strength, cardiorespiratory endurance index, lower body flexibility, and agility and action balance as well as obvious decrease of cholesterol and low-density lipoprotein [4]. Regular exercise not only could enhance the physical fitness of the elderly to have better quality of life, but could also prevent incidence from occurrence.

It is more important for the elderly being able to independently live and safely complete daily activity [5]. For this reason, the elderly exercise prescription design should cover strengths, aerobic endurance, flexibility, agility/dynamic balance, and body mass index as the total performance of human physical and mental characteristic function. Regular physical activity could also strengthen physical fitness. After the 12-week elderly walk training, the walk speed was obviously enhanced and the problem of move with difficulty caused by physical degeneration was also released [6]. Having 50 senior university members be the participants in the 14-week integrated exercise training, including group exercise training, home-based exercise training and health promotion, and exercise management courses, Wei-yu Chang also pointed out the positive improvement of BMI, waistline, heart rate of rest, and functional fitness of the elderly after the integrated exercise training, which could benefit the health promotion of the elderly [7]. As a result, cultivating regular exercise habits, promoting fitness

\footnotetext{
a Corresponding author: vivian@asia.edu.tw
} 
standards, and establishing healthy lifestyles are the primary issues currently.

In addition to regular exercise, it is also important to promote health related physical fitness education and knowledge and instill in favorable healthy lifestyles, expecting to overall promote the health of a community by the active participation of the citizens and the intervention of professional knowledge. Community citizens could also enhance the friendship by the weekly group training and continue the exercise motivation to achieve the national health index. This study aims to discuss the effect of the 12-week regular exercise intervention on the functional fitness of community elderly.

\section{Methodology}

\subsection{Research subject}

Total 25 healthy community elderly (15 females and 10 males) aged above 65 are selected as the research population. In regard to time and manpower limitation, purposive sampling is applied to this study.

\subsection{Experiment procedure}

The participants are proceeded the 24-week fitness exercise training and cognition courses, containing weekly group course and twice-a-week home-based exercise. Functional fitness tests are preceded before and after the intervention. The participants follow the principles of maintaining normal lifestyles, except for the participation in the courses and tests in this study, and do not engage in other exercise. The weekly group course should be fully participated and exercise records need to be filled in to record the physical activity. All examiners in this study have the national fitness examiner certificate approved by Sports Administration, Ministry of Education, Taiwan, in order to ensure the test accuracy.

\subsection{Experiment design}

This study focuses on the integrated exercise training courses, including warm-up, main exercise cool-down, and stretching, aiming to promote distinct fitness, such as stretching exercise, aerobics, strength exercise, and walking, for the intervention training. The $60 \mathrm{~min}$ exercise and $30 \mathrm{~min}$ sports and health cognition courses tend to establish correct sport concept and exercise methods in order to achieve daily work-out habits. ACSM suggested that the elderly should accumulate at lease more than 150 min moderate physical activity every week [8]. The participants in this study maintain daily lifestyles and diet habits during the 12-week exercise intervention, which is practiced three days a week on every other day. The $90 \mathrm{~min}$ exercise training is arranged by the professional exercise instructors on one day, containing 60min gradual and overload strength training 50\% 1RM (10 15 times) and $30 \mathrm{~min}$ low-impact aerobics led by coaches to train physical big muscles. The exercise intensity is up to the rating of perceived exertion (RPE) level 12-15, i.e. at least reaching the moderate exercise intensity of "a bit difficult" [9]. The other two days focus on the independent training of participants, mainly walking, to achieve the moderate exercise intensity of "a bit difficult" requested by the perceived exertion.

\subsection{Test item}

The participants in this study receive functional fitness tests before and after the training. The test items are based on the fitness test proposed by Sports Administration, Ministry of Education, in 2014 to evaluate the daily physical activity ability of the elderly [5]. The evaluation refers to the following 8 test items of body composition, 30sec chair stand (lower limb strength), musculus biceps brachii arm curling (upper limb strength), open-eye single leg standing (static balance), back scratch (upper limb flexibility), chair sitand-reach (lower limb flexibility), chair stand detour (agility/dynamic balance), and $2 \mathrm{~min}$ high knees in place (cardiorespiratory endurance).

\subsection{Data processing}

SPSS20.0 is used for the data analysis, and the basic data and functional fitness of the community elderly are established with Descriptive Statistics. Two-sample $t$ test is applied to analyze the difference before and after the intervention measure, and the research significance is set $\alpha=.05$.

\section{Result}

\subsection{Basic data of participants}

Total 25 healthy community elderly aged above 65 (15 females and 10 males) are selected as the research subjects. The mean ages of female and elderly are $76 \pm 5.33$ and $77 \pm 9.92$, respectively. After the 12-week exercise intervention, the full participation rate reaches above $80 \%$. Table 1 and Table 2 show the pretest/posttest basic data of the male and female elderly, including blood pressure (diastolic pressure, systolic pressure), height $(\mathrm{cm})$, weight $(\mathrm{kg})$, body mass index $(\mathrm{BMI})$, waistline, hipline, and waist-hip ratio. The pretest/posttest analyses show the mean systolic pressure of the male elderly $127.20 \mathrm{mmHg}$ but $126.10 \mathrm{mmHg}$ after the intervention and the mean diastolic pressure $69.10 \mathrm{mmHg}$ but $72.70 \mathrm{mmHg}$ after the intervention, revealing the blood pressure in the normal range. The weight appears $61.49 \mathrm{~kg}$ but $60.90 \mathrm{~kg}$ after the intervention, decreasing $0.59 \mathrm{~kg}$, without significant differences. The body mass index (BMI), waistline, hipline, and waist-hip ratio do not present remarkable changes. The mean systolic pressure of the female elderly shows $122.40 \mathrm{mmHg}$ but $123.00 \mathrm{mmHg}$ after the intervention and the mean diastolic pressure $69.33 \mathrm{mmHg}$ but $70.87 \mathrm{mmHg}$ after the intervention, revealing the blood pressure in the normal range. The weight appears $58.17 \mathrm{~kg}$ but $58.29 \mathrm{~kg}$ after the intervention, 
increasing $0.12 \mathrm{~kg}$, without notable differences. The body mass index (BMI), waistline, and waist-hip ratio do not show remarkable changes, while the hipline achieves significant differences.

Table 1. Pretest/posttest basic data of the male elderly $(n=10)$.

\begin{tabular}{lrrrc}
\hline \multicolumn{1}{c}{ Item } & \multicolumn{1}{c}{ Pretest } & \multicolumn{1}{c}{ Posttest } & \multicolumn{1}{c}{$t$} & \multicolumn{1}{c}{$p$} \\
\hline Systolic pressure & $127.20 \pm 16.06$ & $126.10 \pm 13.92$ & 0.58 & 0.58 \\
Diastolic pressure & $69.10 \pm 7.28$ & $72.70 \pm 10.96$ & -1.46 & 0.18 \\
Height $(\mathrm{cm})$ & $164.80 \pm 7.22$ & $164.50 \pm 7.23$ & 1.00 & 0.34 \\
Weight $(\mathrm{kg})$ & $61.49 \pm 11.38$ & $60.90 \pm 10.82$ & 1.15 & 0.28 \\
BMI & $22.73 \pm 4.42$ & $22.59 \pm 4.26$ & 0.86 & 0.41 \\
Waistline & $89.20 \pm 10.72$ & $86.50 \pm 11.18$ & 1.65 & 0.13 \\
Hipline & $96.55 \pm 7.17$ & $96.50 \pm 6.70$ & 0.05 & 0.97 \\
Waist-hip ratio & $0.92 \pm 0.06$ & $0.89 \pm 0.08$ & 1.34 & 0.21 \\
\hline * $p<.05$ & & & &
\end{tabular}

Table 2. Pretest/posttest basic data of the female elderly ( $n=15)$.

\begin{tabular}{lrrrc}
\hline \multicolumn{1}{c}{ Item } & \multicolumn{1}{c}{ Pretest } & \multicolumn{1}{c}{ Posttest } & \multicolumn{1}{c}{$\mathrm{t}$} & $\mathrm{p}$ \\
\hline Systolic pressure & $122.40 \pm 22.14$ & $123.00 \pm 10.33$ & -0.133 & 0.896 \\
Diastolic pressure & $69.33 \pm 9.96$ & $70.87 \pm 8.09$ & -0.700 & 0.500 \\
Height $(\mathrm{cm})$ & $153.20 \pm 4.72$ & $152.53 \pm 4.03$ & 1.380 & 0.190 \\
Weight $(\mathrm{kg})$ & $58.17 \pm 8.69$ & $58.29 \pm 7.53$ & -0.120 & 0.910 \\
BMI & $24.80 \pm 3.45$ & $28.39 \pm 8.50$ & -1.650 & 0.120 \\
Waistline & $89.50 \pm 9.27$ & $87.93 \pm 9.30$ & 0.990 & 0.340 \\
Hipline & $103.53 \pm 9.38$ & $99.70 \pm 8.50$ & 2.220 & $0.04^{*}$ \\
Waist-hip ratio & $0.87 \pm 0.052$ & $0.88 \pm 0.04$ & -1.060 & 0.310 \\
\hline${ }^{*} p<.05$ & & & &
\end{tabular}

\subsection{Current functional fitness of the elderly}

To understand the current situation based on the fitness test before the intervention, the functional fitness 5-scale norm of seniors in Chiayi City, Taiwan, is referred for the analysis.

(1) Chair stand (evaluation of lower body strength): Females present the mean 16.53 times, with ordinary-favorable level, and males show the mean 15.2 times, with ordinary level.

(2) Musculus biceps brachii left arm curling (evaluation of upper body strength): Females reveal the mean 20.93 times, with good level, and males appear the mean 18.8 times, with favorable level.

(3) Musculus biceps brachii right arm curling (evaluation of upper body strength): Females show the mean 21.4 times, with good level, and males reveal the mean 19.3 times, with favorable-good level.

(4) High knees in place (evaluation of aerobic endurance): Females appear the mean 86.93 times, with ordinary-slightly bad level, and males present the mean 91.9 times, with ordinary-slightly bad level.
(5) Chair sit-and-reach (evaluation of lower body flexibility): Females reveal the mean 0.27 , with slightly bad-bad level, and males appear the mean 3.6, with slightly bad level.

(6) Open-eye single leg standing (evaluation of balance): Female present the mean $12.78 \mathrm{sec}$, with ordinary-favorable level, and males show the mean $21.09 \mathrm{sec}$, with favorable-good level.

(7) Left back scratch (evaluation of upper body flexibility): Females appear the mean -13.93 , with slightly bad-bad level, and males present the mean 16.6, with slightly bad-bad level.

(8) Right back scratch (evaluation of upper body flexibility): Females show the mean -4.93 , with ordinary-slightly bad level, and males reveal the mean -13 with ordinary-slightly bad level.

(9) Chair stand detour (evaluation agility and dynamic balance): Females appear the mean $6.88 \mathrm{sec}$, with ordinary level, and males present the mean $6.26 \mathrm{sec}$, with ordinary-favorable level.

\subsection{Effects of intervention on functional fitness}

Table 3 shows that the male elderly reveal notable differences on the upper limb strength (musculus biceps brachii left arm curling, musculus biceps brachii right arm curling), the lower limb strength (chair stand) does not reach the significance, and the static balance (openeye single leg standing) and agility and dynamic balance (chair stand detour) achieve remarkable differences $(p<$ $0.5)$. In regard to flexibility, the upper limb flexibility (back scratch), lower limb flexibility (chair sit-andreach), and cardiorespiratory endurance (high knees in place) do not reach significant differences.

Table 3. Male functional fitness pretest/posttest difference $(n=10)$

\begin{tabular}{ccccc}
\hline Item & Pretest & Posttest & $t$ & $p$ \\
\hline Chair stand & $15.20 \pm 3.74$ & $17.42 \pm 4.91$ & -1.46 & 0.1790 \\
$\begin{array}{c}\text { Musculus biceps brachii } \\
\text { arm curling (left) }\end{array}$ & $18.80 \pm 2.78$ & $21.20 \pm 3.19$ & 4.27 & $0.002^{*}$ \\
$\begin{array}{c}\text { Musculus biceps brachii } \\
\text { arm curling (right) }\end{array}$ & $19.30 \pm 3.65$ & $22.10 \pm 3.07$ & -5.25 & $0.001^{*}$ \\
High knees in place & $91.90 \pm 12.82$ & $94.90 \pm 10.86$ & -2.13 & 0.0620 \\
$\begin{array}{c}\text { Chair sit-and-reach } \\
\text { Open-eye single leg } \\
\text { standing }\end{array}$ & $15.20 \pm 3.74$ & $17.42 \pm 4.91$ & -0.72 & 0.4870 \\
Back scratch (left) & $-16.60 \pm 14.42$ & $-17.90 \pm 8.19$ & 0.33 & 0.7500 \\
Back scratch (right) & $-13.00 \pm 10.32$ & $-11.60 \pm 10.76$ & -2.26 & 0.0500 \\
Chair stand detour & $15.20 \pm 3.74$ & $17.42 \pm 4.91$ & 2.99 & $0.015^{*}$ \\
\hline$* p<.05$ & & & &
\end{tabular}

Table 4 reveals that the female elderly present remarkable differences on the upper limb strength (musculus biceps brachii left arm curling, musculus biceps brachii right arm curling), the lower limb strength 
(chair stand) does not achieve the significance, and the cardiorespiratory endurance (high knees in place), static balance (open-eye single leg standing), and agility and dynamic balance(chair stand detour) reach notable differences $(p<0.5)$. In terms of flexibility, both upper limb flexibility (back scratch) and lower limb flexibility (chair sit-and-reach) do not achieve significant differences.

Table 4. Female functional fitness pretest/posttest difference $(n=15)$

\begin{tabular}{|c|c|c|c|c|}
\hline Item & Pretest & Posttest & $t$ & $p$ \\
\hline Chair stand & $16.53 \pm 1.36$ & $16.87 \pm 4.58$ & -0.310 & 0.7600 \\
\hline $\begin{array}{l}\text { Musculus biceps brachii } \\
\text { arm curling (left) }\end{array}$ & $20.93 \pm 3.06$ & $22.39 \pm 3.97$ & -2.940 & $0.011 *$ \\
\hline $\begin{array}{l}\text { Musculus biceps brachii } \\
\text { arm curling (right) }\end{array}$ & $21.40 \pm 4.47$ & $23.93 \pm 4.59$ & -4.670 & $.000^{*}$ \\
\hline High knees in place & $86.93 \pm 12.12$ & $93.20 \pm 15.92$ & -3.010 & $0.009^{*}$ \\
\hline Chair sit-and-reach & $0.27 \pm 5.82$ & $1.53 \pm 6.84$ & -0.670 & 0.5170 \\
\hline $\begin{array}{c}\text { Open-eye single leg } \\
\text { standing }\end{array}$ & $12.78 \pm 16.75$ & $14.27 \pm 16.60$ & -2.280 & $0.039 *$ \\
\hline Back scratch (left) & $-13.93 \pm 10.07$ & $-11.87 \pm 8.19$ & -1.050 & 0.3110 \\
\hline Back scratch (right) & $-4.93 \pm 7.47$ & $-3.80 \pm 7.18$ & -1.800 & 0.0940 \\
\hline Chair stand detour & $6.88 \pm 0.90$ & $6.34 \pm 0.92$ & 4.232 & $0.001 *$ \\
\hline
\end{tabular}

\section{Conclusion}

Senior Fitness Test (SFT) is utilized for evaluating the functional fitness of the elderly in this study. The research findings show remarkable improvement of the functional fitness of the female elderly, including upper limb strength, cardiorespiratory endurance, and balance, after the intervention of fitness exercise training and cognition courses. Although flexibility does not reach the significance, it reveals progress, comparing to the performance before exercise intervention. The result conforms to the research of Tai-yu Huang, Che-jung Chang, Mang-hui Lin \& Chin-lung Fang [10]. In their research, 35 female elderly aged above 60 were proceeded 10-week water aerobic training, in which flexibility was improved but did not achieve the significance, while the rest functional fitness of upper/lower limb strength, aerobic endurance, and static and dynamic balance appeared notable improvement. Besides, the daily physical activity ability was promoted and the degeneration of physiological function resulted from increasing ages slowed down. Hallage et al. pointed out the remarkable effect of the 12-week aerobics intervention on the body mass index (BMI), waistline, chair stand, musculus biceps brachii arm curling, high knees in place, chair sit-and-reach, open-eye single leg standing, back scratch, and chair stand detour of female elderly [11]. Accordingly, aerobics could obviously improve the functional fitness of female elderly and was regarded as an effective training to prevent fitness from loss.

Regarding the male elderly, the results reveal the remarkable improvement of upper limb strength and balance. A one-year health promotion program for 148 elderly showed that aerobic and strength training and health education program and control could improve aerobic endurance and dynamic balance and promote fitness and health conditions [12]. Although lower limb strength, flexibility, and cardiorespiratory endurance did not achieve the significance, they presented progress, comparing to those before the intervention. Jhen-Yi Chen, Yen-Ju Lin, Yu-Ching Weng \& Su-Chuan Yuan discussed the effect of the 12-week regular walking intervention on the fitness of community elderly with chronic diseases [13]. The results showed that, in addition to the promotion of cardiopulmonary function, the fitness of the elderly with chronic diseases was actually improved after exercise intervention. They suggested adding walking in the exercise intervention, as it was life -oriented exercise with better motivation to continue, in order to present more notable benefits on cardiopulmonary function.

From above research results, exercise intervention not only promotes the functional fitness of the elderly but also improves the health conditions. By comparing the physical activity of the elderly [14], Kim, Dogra, AlSahab \& Tamim preceded the 16-week Taijiquan intervention in the elderly with and without fitness experiences. The results revealed that the elderly without experiences appeared remarkable differences on functional fitness after the intervention measure. The above statement is therefore proven. Health promotion could have the elderly present better confidence on exercise or daily activity. Aiming at intervening the daily life of weak elderly with exercise training program, Chou, Hwang \& Wu [15] and Shimada, Uchiyama \& Kakurai [16] revealed no significant effect of exercise intervention on the quality of life in groups, but increasing walk speed and improved balance to promote the daily activity performance. Consequently, the practice of exercise intervention programs in the elderly presents the benefits to enhance physical fitness, strengthen body, and promote independent living abilities for more active and safe aged life.

\section{Acknowledgement}

We are grateful for the project funding support of Ministry of Science and Technology (MOST 104-2629E-468-002) and would like to show our appreciation.

\section{References}

1. National Development Council of the Executive Yuan of Taiwan, The report of Republic of China from 2012 to 2060 census (National Development Council of the Executive Yuan, Taiwan, 2014)

2. S. Roberts, R.A. Robergs, P.G. Hanson, Clinical exercise testing and prescription: Theory and 
application (CRC Press, Boston, Massachusetts, 1997)

3. C.L. Liu, J.Y. Hou, R.H. Tu, J. of National Pingtung University Physical Education Research 17, 139-144 (2014)

4. P.C. Chung, F.Y. Tseng, Formosan Journal of Medicine 13(2), 133-140 (2009)

5. C.J. Jones, R.E. Rikli, Journal of Aging and Physical Activity 7, 129-161 (1999)

6. J.M. VanSwearingen, S. Perera, J.S. Brach, D. Wert, S.A. Studenski, Physical Therapy 91(12), 1740-1751 (2011)

7. W.Y. Chang, J. of National Taichung University of Science and Technology Physical Education Research 10, 45-54 (2014)

8. American College of Sports Medicine, ACSM's guidelines for exercise testing and prescription (8th ed.), (American College of Sports Medicine, Philadelphia, PA, 2010)

9. G. Borg, Scandinavian Journal of Work, Environment \& Health 16, 55-58 (1990)

10. T.Y. Huang, C.J. Chang, M.H. Nin, C.L. Fang, Journal of Exercise Physiology and Fitness 3, 121129 (2005)

11. T. Hallage, M.P. Krause, L. Haile, C.P. Miculis, E.F. Nagle, R.S. Reis, S.G. Da Silva, Journal of Strength and Conditioning Research 24(8), 2261-2266 (2010)

12. Obesity, fitness \& wellness week, Studies from University of Porto Add New Findings in the Area of Physical Fitness (NewsRx.com, Atlanta, GA, July 30 2011)

13. J.Y. Chen, Y.J. Lin, Y.C. Weng, S.C. Yuan, Cheng Ching Medical Journal 11(1), 39-48 (2015)

14. T.H. Kim, S. Eke Dogra, B. Al-Sahab, H. Tamim, Alternative Therapies in Health and Medicine 20(3), 20-25 (2014)

15. C.H. Chou, C.L. Hwang, Y.T. Wu, Arch. Phys. Med. Rehabil. 93(2), 237-244 (2012)

16. H. Shimada, Y. Uchiyama, S. Kakurai, Clinical Rehabilitation 17(5), 472-479 (2003) 